\author{
Michalina Kołodziejczak ${ }^{1,2}$, Eliano Pio Navarese ${ }^{1,2,3,4}$, Jacek Kubica ${ }^{1}$ \\ ${ }^{1}$ Department of Cardiology and Internal Medicine, Collegium Medicum, Nicolaus Copernicus University, Bydgoszcz, Poland \\ 2 Systematic Investigation and Research on Interventions and Outcomes (SIRIO) MEDICINE Cardiovascular Research Network \\ ${ }^{3}$ Interventional Cardiology and Cardiovascular Medicine Research, Cardiovascular Institute, Mater Dei Hospital, Bari, Italy \\ ${ }^{4}$ Faculty of Medicine, University of Alberta, Edmonton, Canada
}

\title{
Rationale and design of PREvalence of DyspneA in patients treated with TicagreIOR (PREDATOR) program
}

\author{
Corresponding author: \\ Corresponding author: \\ Michalina Kołodziejczak \\ Department of Cardiology and Internal \\ Medicine, Collegium Medicum, \\ Nicolaus Copernicus University, \\ Bydgoszcz, Poland, \\ e-mail: \\ kolodziejczak.michalina@gmail.com
}

\begin{abstract}
Background: Ticagrelor, a reversible P2Y12 inhibitor, is a mainstay of antiplatelet strategy in patients with acute coronary syndrome (ACS). However, a large number of ticagrelor-induced dyspnea decrease patients' adherence and reduce an overall efficacy of the therapy.

Design: The PREDATOR program consists of phase III and IV, multicenter, randomized, double-blind, placebo-controlled clinical trials and preceding pilot studies that assesses the prevalence and treatment of ticagrelor-induced dyspnea in coronary artery disease (CAD) and ACS patients. The PREDATOR LD is designed to evaluate the occurrence of dyspnea after $180 \mathrm{mg}$ ticagrelor loading dose, and relief of dyspnea by theophylline administration in low-to-high risk acute coronary syndromes without ST-segment elevation (NSTE-ACS) and stable CAD designated to undergo invasive treatment. The PREDATOR MD is a cross-over trial in stable CAD patients 1 year after percutaneous coronary intervention for ACS. Enrolled patients will be randomized to one of four antiplatelet treatment regimens (ticagrelor 2x90 mg, ticagrelor 2x60 mg, ticagrelor 2x45 mg or clopidogrel 75 mg [morning] + placebo [evening]) or placebo and will be assessed for dyspnea at the day 7, then undergo a switch of treatment and reassessment at day 14. The sample size will be estimated based on preceding pilot studies.

Discussion: The PREDATOR LD is expected to prospectively assess dyspnea rate with a loading dose of ticagrelor, and analyze a potential of theophylline to elevate symptoms of ticagrelor-induced dyspnea, while the PREDATOR MD will prospectively assess dyspnea and adverse events rate with a maintenance dose of P2Y12 inhibitors prospectively assess. All evaluations will be conducted using standardized metrics for dyspnea quantification.

Key words: antiplatelet, ticagrelor, dyspnea, loading dose, maintenance dose, rationale, trial
\end{abstract}

Med Res J 2018; 3 (4): 215-220

\section{Introduction}

Antiplatelet therapy with P2Y12 inhibitors is a mainstay strategy for the management of acute coronary syndrome (ACS) patients. Ticagrelor, novel and reversible P2Y12 inhibitor, is recommended as a class I therapy for 12 months after an ACS event by the European Society of Cardiology and the American College of Cardiology/American Heart Association guideline committees [1-3]. The optimal antiplatelet effect prevents from thrombotic complications, including devastating in consequences stent thrombosis that drives cardiovascular mortality in the early stage post percutaneous coronary intervention (PCI) [4-7]. The potency of antithrombot- ic strategy with novel P2Y12 inhibitor, ticargelor, as compared to its irreversible counterpart clopidogrel, is well established [8-10]. In large randomized controlled trial (RCT) ticagrelor (90 mg or $60 \mathrm{mg}$, twice daily) in combination with aspirin significantly reduced the risk of cardiovascular death, myocardial infarction (MI), or stroke as compared with clopidogrel in patients who had prior MI 1-3 years earlier [11]. Bleeding and respiratory adverse events, however, were more frequently observed with ticagrelor use.

One of the reasons of ineffectiveness of antiplatelet therapy originates from low adherence and discontinuation of the therapy due to adverse events [12-17]. Dyspnea is a relatively frequent adverse event ob- 
served during ticagrelor therapy, and while it is usually reported as "mild" or "moderate" in intensity among patients experiencing it, it remains an overt reason for discontinuation of the assigned treatment [18]. In the secondary analysis of the PEGASUS-TIMI 54 Trial the rate of discontinuation due to an adverse event was the highest in the treatment arm receiving $90 \mathrm{mg}$ of ticagrelor (19\%), followed by the treatment arm receiving $60 \mathrm{mg}$ of ticagrelor (16\%), both of which were significantly higher than that in the placebo arm (9\%), with the differences most marked within the first year from randomization [19]. A dose-dependent relative increase of both dyspnea and dyspnea requiring discontinuation was present, however, the discontinuation rate resulting from dyspnea among those subjects, who reported dyspnea, was 5-flod higher than observed in the PLATO trial. Interestingly, discontinuation due to dyspnea $(6.2 \%$ ticagrelor $90 \mathrm{mg}$ bid vs $4.3 \%$ ticagrelor $60 \mathrm{mg}$ bid vs $0.7 \%$ placebo) had the same prevalence as the disocntinuation due to bleeding (6.5\% ticagrelor $90 \mathrm{mg}$ bid vs $5.1 \%$ ticagrelor $60 \mathrm{mg}$ bid vs $1.2 \%$ placebo). The increased risk of bleeding is on the other side of spectrum of thrombotic milieu and is inevitable in successful antithrombotic management; dyspnea, however, remains an adverse event with a high potential of prevention [14]. Ticagrelor-induced dyspnea exerts generally no effort on the patient's activities of daily life and can be resolved quickly, as it does not influence the patient's cardiac nor pulmonary function. Taking together not sufficient awareness of the recognition and management of ticagrelor-related dyspnea, especially among elderly ACS patients treated with several drugs, there exists a global underestimation and mismanagement of this adverse event [20]. For a proper management of patients complaining of dyspnea when taking ticagrelor, all clinically plausible causes of dyspnea must be considered before attributing it to medication itself [21]. Episodes of dyspnea tend to occur during first days after the initiation of the therapy [18]. Dyspnea may have devastating effects when leading to treatment discontinuation, especially in the case of frail population as ACS patients. In the first multicenter observational prospective study to investigate rate of ticagrelor-related dyspnea and its impact on daily life in patients in their first month post $\mathrm{PCl}$, ticagrelor was withdrawn in $16.7 \%$ patients during the $1^{\text {st }}$ month of follow-up, with ticagrelor-related dyspnea at the rate of $55.6 \%$ of all withdrawal reasons [22]. To provide further optimization of the antiplatelet strategies it is mandatory to avoid discontinuation due to a preventable adverse event.

A number of potential mechanisms could cause ticagrelor-induced dyspnea. It has been hypothesized that the sensation of dyspnea is related to reversibility of the P2Y12 inhibiting agent origins from increased levels of adenosine, which cellural reuptake is impaired [23].
Pulmonary vagal $C$ fibres are stimulated by increased adenosine levels through the $A_{1} R$ and $A_{2 A} R$ receptors on vagal sensory $C$ fibers, which mediate the sensation of dyspnea. The increase in serum adenosine is higher with reversible (i.e. ticagrelor) rather than irreversible P2Y12 inhibitors (clopidogrel) [24]. Therefore, potential treatment options for ticagrelor-associated dyspnea may include adenosine antagonism, such as aminophylline or theophylline. The cases of successful reversal of ticagrelor-induced dyspnea have been described in literature [25]. Their role is still not yet fully addressed in a large clinical study. In a promising randomized trial TROCADERO (TRial Of Caffeine to Alleviate DyspnEa Related to ticagrelOr) the primary objective was to assess the efficacy of caffeine in reducing symptoms of ticagrelor-induced dyspnea, measured by the visual analog scale area under the curve (VAS AUC), in patients after an ACS [26]. However, out of 514 subjects who participated in the questionnaire survey, only a small number of patients experienced dyspnea that required an intervention and were randomized to one of the strategies: 13 to caffeine, and 10 to placebo. In this context, dyspnea and treatment to evelate its symptoms were not prospectively assessed in a large study with a use of standardized tool, thus, its prevalence and severity is not well established.

In the PREDATOR program, we aim for the first time to 1) prospectively assess dyspnea rate with a loading dose of reversible P2Y12 inhibitor, ticagrelor, 2) analyze a potential of theophylline to elevate symptoms of ticagrelor-induced dyspnea, 3) prospectively assess dyspnea and adverse events rate with a maintenance dose of P2Y12 inhibitors. All evaluations will be conducted using standardized metrics for dyspnea quantification.

\section{Methods}

\section{Program design and objectives}

The PREvalence of DyspneA in patients treated with TicagrelOR (PREDATOR) is an international, multicenter program consisting of two trials preceded by pilot studies (Figure 1). The inclusion and exclusion criteria for both PREDATOR Loading Dose (LD) Study and PREDATOR Maintenance Dose (MD) Study are presented in Table 1. The same criteria will apply to the pilot studies. The main objective of the program is to prospectively and quantitatively assess, with the use of a standardized score, prevalence of dyspnea with ticargelor and a potential of theophylline administration on dyspnea cessation among ACS and stable CAD patients. The study will be conducted in accordance with the principles contained in the Dec- 
laration of Helsinki, and every study site will receive an approval from the Local Ethics Committee. Each patient will provide a written informed consent to participate in the study.

\section{Statistical design and analysis}

Since the rate of dyspnea with ticagrelor use varies in definitions and rates across previously published literature, we decided to perform preceding pilot studies to estimate the sample size.

\section{PREDATOR LD}

The PREDATOR LD is a double-blind, placebo-controlled, randomized trial in low-to-high risk acute coronary syndromes without ST-segment elevation

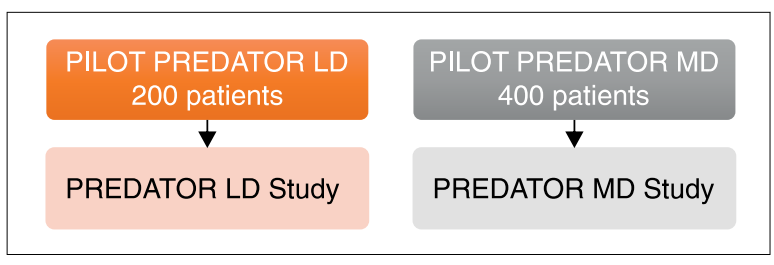

Figure 1. Schematic design of the PREDATOR program
(NSTE-ACS) and stable coronary disease designated to undergo invasive treatment. The primary objective of the study is to evaluate the occurrence of dyspnea after $180 \mathrm{mg}$ ticagrelor loading dose, with following secondary objective of assessing relief of dyspnea by theophylline administration. Patients will undergo two stages of randomization - first randomization to $180 \mathrm{mg}$ ticagrelor LD vs. placebo followed by a switch of therapy after 2 hours in patients without dyspnea, and second randomization to theophylline p.o. (loading dose $5 \mathrm{mg} / \mathrm{kg}$, maintenance dose $100 \mathrm{mg}$ tid) vs. placebo p.o. of only patients with dyspnea in 1st randomization stage (Figure 2). The study follow-up will be stratified to three durations, driven by randomization: 1) $1^{\text {st }}$ follow-up, within 2 hours after first randomization (patients with dyspnea directly undergo second randomization, while patients without dyspnea by the end of follow-up have the allocated therapy switched), 2) $2^{\text {nd }}$ follow-up, within 2 hours after the $1^{\text {st }}$ follow-up, up to 4 hours after first randomization (patients with dyspnea undergo second randomization, while patients without dyspnea undergo coronary angiography/PCI), 3) $3^{\text {rd }}$ follow-up, within 1 hour after second randomization, and up to 5 hours after first randomization (all patients undergo coronary angiography/PCl, while for patients in whom dyspnea have occurred at first follow-up period the study treatment will be unblinded after termination of the protocol and before $\mathrm{PCl}$; patients in placebo arm

Table 1. Inclusion and exclusion criteria of PREDATOR LD Study and PREDATOR MD Study

\begin{tabular}{|c|c|c|}
\hline & PREDATOR LD Study & PREDATOR MD Study \\
\hline \multirow[t]{2}{*}{$\begin{array}{l}\text { Inclusion } \\
\text { criteria }\end{array}$} & $\begin{array}{l}\text { - diagnosis of low, intermediate or high risk NSTE-ACS } \\
\text { designated to invasive treatment or diagnosis of stable } \\
\text { coronary disease designated to invasive treatment }\end{array}$ & $\begin{array}{l}\text { - diagnosis of stable coronary disease } 1 \text { year after } \mathrm{PCI} \\
\text { for acute coronary syndrome with known anatomy of } \\
\text { coronary arteries designated to invasive treatment }\end{array}$ \\
\hline & \multicolumn{2}{|c|}{$\begin{array}{l}\cdot \text { male or non-pregnant female } \\
\qquad \text { age }>18 \text { years old } \\
\text { of informed consent for angiography and PCI } \\
\text { rritten informed consent to participate in the study }\end{array}$} \\
\hline \multirow[t]{2}{*}{$\begin{array}{l}\text { Exclusion } \\
\text { criteria }\end{array}$} & $\begin{array}{l}\text { - diagnosis of STEMI } \\
\text { - diagnosis of very high risk NSTE-ACS designated to } \\
\text { immediate invasive treatment } \\
\text { - dyspnea present at screening } \\
\text { - contraindications for ticagrelor or theophylline } \\
\text { - second or third degree atrioventricular block during } \\
\text { screening for eligibility }\end{array}$ & $\begin{array}{l}\text { - diagnosis of acute coronary syndrome } \\
\text { - PCl with coronary stenting during the last } 12 \text { months } \\
\text { - chronic dyspnea present at screening (e.g. due to } \\
\text { severe COPD) } \\
\text { - contraindications for ticagrelor, prasugrel, clopidogrel } \\
\text { - history of second or third degree atrioventricular block } \\
\text { without implanted pacemaker }\end{array}$ \\
\hline & \multicolumn{2}{|c|}{$\begin{array}{c}\text { - treatment with ticagrelor or theophylline within } 14 \text { days before the study enrolment } \\
\text { - current treatment with oral anticoagulant or chronic therapy with low-molecular-weight heparin } \\
\cdot \text { active bleeding } \\
\cdot \text { history of intracranial haemorrhage } \\
\cdot \text { recent gastrointestinal bleeding (within } 30 \text { days) } \\
\cdot \text { history of coagulation disorders } \\
\cdot \text { history of major surgery or severe trauma (within } 3 \text { months) } \\
\cdot \text { patient required dialysis } \\
\cdot \text { manifest infection or inflammatory state } \\
\cdot \text { respiratory failure } \\
\text { - current therapy with strong CYP3A inhibitors or strong CYP3A inducers }\end{array}$} \\
\hline
\end{tabular}




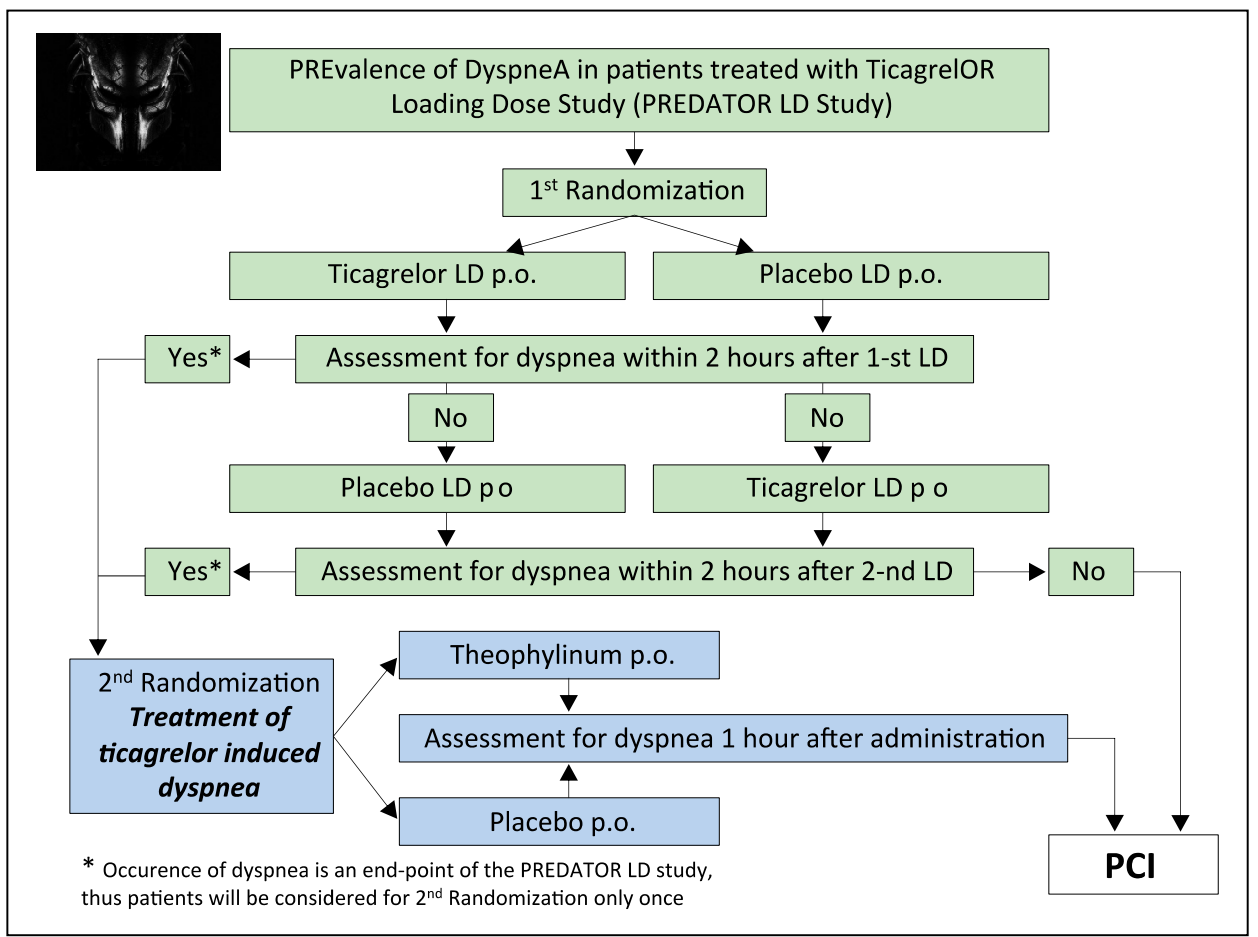

Figure 2. PREDATOR LT Study design

will unblindly receive ticagrelor LD). Dyspnea, as well as the relief from it, will be quantified with the Modified Borg Dyspnoea Score (Table 2). The exact number of study population will be determined based on the PREDATOR LD pilot study including 200 patients.

\section{PREDATOR MD}

The PREDATOR MD is a double-blind, placebo-controlled, cross-over, randomized trial conducted in stable coronary artery disease (CAD) patients 1 year after $\mathrm{PCI}$ for ACS. Enrolled patients will be randomized to one of four antiplatelet treatment regimens (ticagrelor 2x90 mg or ticagrelor $2 \times 60 \mathrm{mg}$ or ticagrelor $2 \times 45 \mathrm{mg}$ or clopidogrel $75 \mathrm{mg}$ [morning] + placebo [evening]) or placebo (1:1:1:1:1 ratio), and will be assessed for dyspnea at the day 7 of the allocated therapy. On the same day all patients will undergo a switch of treatment and will be reassessed for dyspnea at day 14 . The primary objective of the study is to evaluate the occurrence of dyspnea with different maintenance dose of antiplatelet treatment regimens. Dyspnea will be quantified with the Modified Borg Dyspnoea Score. The secondary endpoints of this study, evaluating the safety of P2Y12 inhibiting therapy, include major bleedings according to the Thrombolysis In Myocardial Infarction (TIMI) criteria, minor bleedings according to TIMI criteria, clinically relevant bradycardia, level of serum uric acid, neutrophil count, overall premature discontinuation of the study drug and discontinua-
Table 2. Modified Borg Dyspnoea Scale

\begin{tabular}{ll}
\hline Rate & Symptoms \\
\hline 0 & Nothing at all \\
0.5 & Very, very slight (just noticeable) \\
1 & Very slight \\
2 & Slight \\
3 & Moderate \\
4 & Somewhat severe \\
5 & Severe \\
6 & \\
7 & Very severe \\
8 & \\
9 & Very, very severe (almost maximal) \\
10 & Maximal \\
\hline
\end{tabular}

tion of the study drug due to dyspnoe. Discontinuation is defined as study drug cessation following medical contact. The exact number of study population will be determined based on the PREDATOR MD pilot study including 400 patients.

\section{Study organization}

The executive committee is responsible for the overall design, conduct, and supervision of the study, 


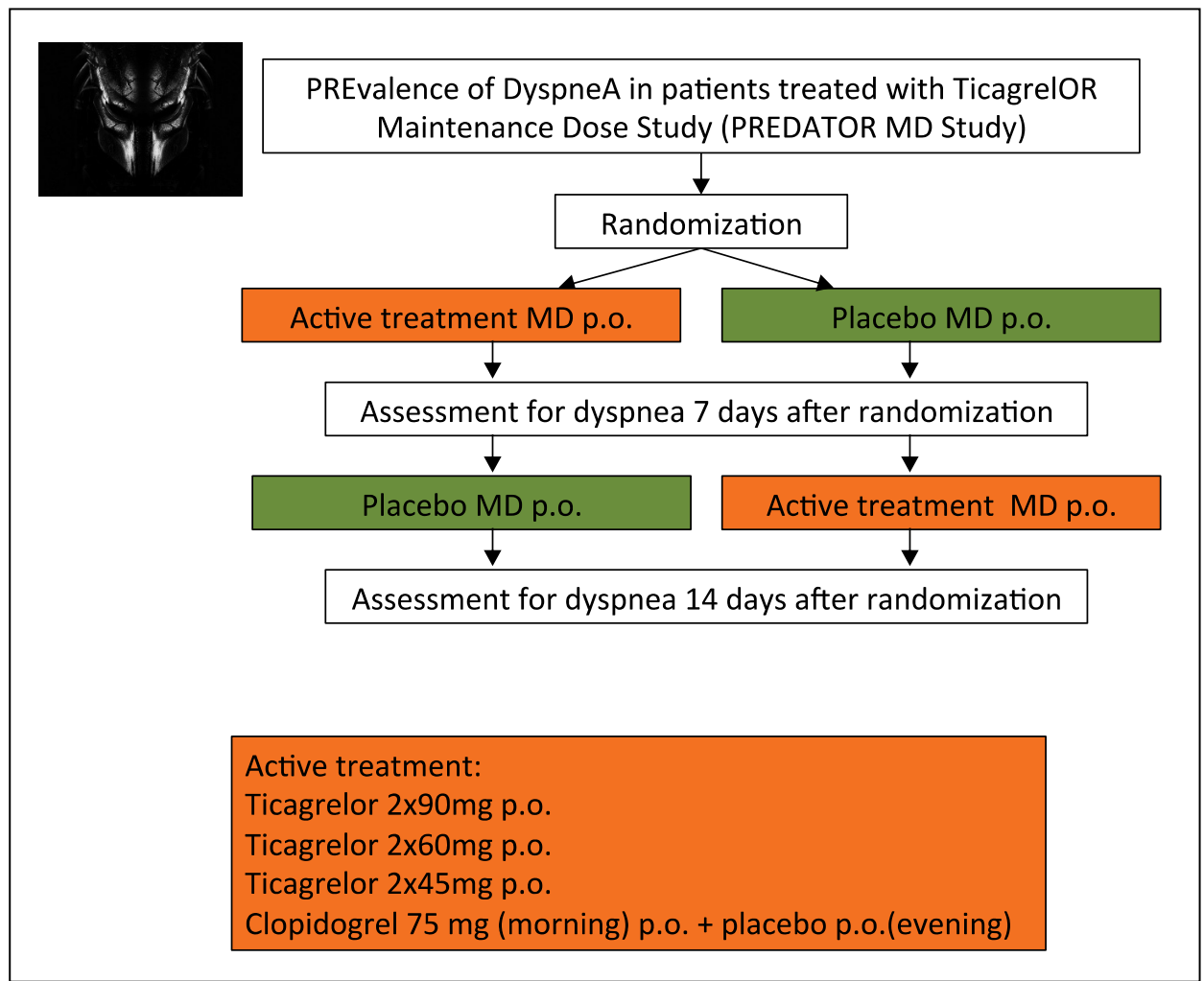

Figure 3. PREDATOR MD Study design

including the development of any protocol amendments. The independent academic statistician will perform or confirm all statistical analyses of the final data. A National Leaders Committee, composed of lead investigators from each participating country, works in tandem with the Executive Committee. The executive committee is composed of representatives from the Collegium Medicum of Nicolaus Copernicus University (Bydgoszcz, Poland), and other collaborating centers. A clinical events committee reviews and adjudicates each suspected clinical end point event in a blinded fashion.

\section{Conclusions}

The PREDATOR program includes phase III and IV, multicenter, randomized, double-blind, placebo-controlled clinical trials that assesses the prevalence and treatment of ticagrelor-induced dyspnea in CAD/ACS patients. Those trials will provide important information regarding the impact of ticagrelor loading and maintenance dose on the occurrence and severity of dyspnea, prospectively assessed by a standardized score. A disclosure of such information could provide medical practitioners with more effective tools preventing from consequences of therapy discontinuation due to mild adverse events.

\section{Acknowledgements}

The PREDATOR program is funded by Collegium Medicum of Nicolaus Copernicus University and did not receive any external funding.

\section{Statement of competing of interests}

Jacek Kubica received a consulting fee from AstraZeneca. Eliano Pio Navarese received honoraria from Astra Zeneca, Sanofi-Regeneron, Eli-Lilly and grants from Amgen. All other authors have reported no relationships relevant to the contents of this paper that could be construed as a conflict of interest.

The publication was supported by AstraZeneca Pharma Poland sp. o.o.

\section{List of abbreviations}

ACS - acute coronary syndrome, CAD - coronary artery disease, $\mathrm{LD}$ - loading dose, MD - maintenance dose, $\mathrm{MI}$ - myocardial infarction, NSTE-ACS - acute coronary syndromes without ST-segment elevation, $\mathrm{PCl}$ - percutaneous coronary intervention, $\mathrm{RCT}$ - randomized controlled trial, STEMI — ST-segment elevation 


\section{myocardial infarction, TIMI - Thrombolysis In Myocardial Infarction, VAS AUC - visual analog scale area under the curve}

\section{References}

1. Neumann FJ, Sousa-Uva M, Ahlsson A, et al. ESC Scientific Document Group . 2018 ESC/EACTS Guidelines on myocardial revascularization. Eur Heart J. 2018 [Epub ahead of print], doi: 10.1093/eurheartj/ehy394, indexed in Pubmed: 30165437

2. O'Gara P, Kushner F, Ascheim D, et al. 2013 ACCF/AHA Guideline for the Management of ST-Elevation Myocardial Infarction: Executive Summary. Circulation. 2013; 127(4): 529-555, doi 10.1161/cir.0b013e3182742c84.

3. Amsterdam EA, Wenger NK, Brindis RG, et al. ACC/AHA Task Force Members. 2014 AHA/ACC guideline for the management of patients with non-ST-elevation acute coronary syndromes: a report of the American College of Cardiology/American Heart Association Task Force on Practice Guidelines. Circulation. 2014; 130(25) e344-e426, doi: 10.1161/CIR.0000000000000134, indexed in Pubmed: 25249585

4. Byrne RA, Joner M, Kastrati A. Stent thrombosis and restenosis: what have we learned and where are we going? The Andreas Grüntzig Lecture ESC 2014. Eur Heart J. 2015; 36(47): 3320-3331, doi: 10.1093/eurheartj/ehv511, indexed in Pubmed: 26417060.

5. Adamski P, Sikora J, Laskowska E, et al. Comparison of bioavailability and antiplatelet action of ticagrelor in patients with ST-elevation myocardial infarction and non-ST-elevation myocardial infarction: A prospective, observational, single-centre study. PLoS One. 2017 12(10): e0186013, doi: 10.1371/journal.pone.0186013, indexed in Pubmed: 29023473.

6. Adamski P, Ostrowska M, Sikora J, et al. Comparison of Ticagrelor Pharmacokinetics and Pharmacodynamics in STEMI and NSTEM Patients (PINPOINT): protocol for a prospective, observational, single-centre study. BMJ Open. 2017; 7(4): e013218, doi: 10.1136/bmjopen-2016-013218, indexed in Pubmed: 28446521.

7. Adamski P, Adamska U, Ostrowska M, et al. New directions for pharmacotherapy in the treatment of acute coronary syndrome. Expert Opin Pharmacother. 2016; 17(17): 2291-2306, doi 10.1080/14656566.2016.1241234, indexed in Pubmed: 27677394.

8. Adamski P, Buszko K. Sikora J, et al. Metabolism of ticagrelor in patients with acute coronary syndromes. Sci Rep. 2018; 8(1): 11746 doi: 10.1038/s41598-018-29619-9, indexed in Pubmed: 30082687

9. Wallentin L, Becker RC, Budaj A, et al. PLATO Investigators. Ticagrelor versus clopidogrel in patients with acute coronary syndromes. N Engl J Med. 2009; 361(11): 1045-1057, doi: 10.1056/NEJMoa0904327, indexed in Pubmed: 19717846.

10. Adamski P, Koziński M, Ostrowska M, et al. Overview of pleiotropic effects of platelet P2Y12 receptor inhibitors. Thromb Haemost. 2014 112(2): 224-242, doi: 10.1160/TH13-11-0915, indexed in Pubmed: 24763899.

11. Bonaca MP, Bhatt DL, Cohen M, et al. PEGASUS-TIMI 54 Steering Committee and Investigators. Long-term use of ticagrelor in patients with prior myocardial infarction. N Engl J Med. 2015; 372(19): 1791-1800 doi: 10.1056/NEJMoa1500857, indexed in Pubmed: 25773268.

12. Kubica A, Kosobucka A, Fabiszak T, et al. Assessment of adherence to medication in patients after myocardial infarction treated with percutaneous coronary intervention. Is there a place for newself-reported questionnaires? Curr Med Res Opin. 2018 [Epub ahead of print]:
1-9, doi: 10.1080/03007995.2018.1510385, indexed in Pubmed: 30091642.

13. Kosobucka A, Michalski P, Pietrzykowski $€$, et al. Adherence to treatment assessed with the Adherence in Chronic Diseases Scale in patients after myocardial infarction. Patient Prefer Adherence. 2018; 12 : 333-340, doi: 10.2147/PPA.S150435, indexed in Pubmed: 29551891.

14. Kubica J, Adamski P, Buszko K, et al. Rationale and Design of the Effectiveness of LowEr maintenanCe dose of TicagRelor early After myocardial infarction (ELECTRA) pilot study. Eur Heart J Cardiovasc Pharmacother. 2018; 4(3): 152-157, doi: 10.1093/ehjcvp/pvx032, indexed in Pubmed: 29040445.

15. Kubica A, Obońska K, Fabiszak T, et al. Adherence to antiplatelet treatment with P2Y12 receptor inhibitors. Is there anything we can do to improve it? A systematic review of randomized trials. Curr Med Res Opin. 2016; 32(8): 1441-1451, doi: 10.1080/03007995.2016.1182901, indexed in Pubmed: 27112628

16. Kubica A Kasprzak M, Obońska K, et al. Discrepancies in assessment of adherence to antiplatelet treatment after myocardial infarction. Pharmacology. 2015; 95(1-2): 50-58, doi: 10.1159/000371392, indexed in Pubmed: 25592409

17. Kubica A, Kasprzak M, Siller-Matula J, et al. Time-related changes in determinants of antiplatelet effect of clopidogrel in patients after myocardial infarction. Eur J Pharmacol. 2014; 742: 47-54, doi: 10.1016/j. ejphar.2014.08.009, indexed in Pubmed: 25199965.

18. Storey RF, Becker RC, Harrington RA, et al. Characterization of dyspnoea in PLATO study patients treated with ticagrelor or clopidogrel and its association with clinical outcomes. Eur Heart J. 2011; 32(23): 2945-2953, doi: 10.1093/eurheartj/ehr231, indexed in Pubmed: 21804104.

19. Bonaca MP, Bhatt DL, Oude Ophuis T, et al. Long-term Tolerability of Ticagrelor for the Secondary Prevention of Major Adverse Cardiovascular Events: A Secondary Analysis of the PEGASUS-TIMI 54 Trial. JAMA Cardiol. 2016; 1(4): 425-432, doi: 10.1001/jamacardio.2016.1017, indexed in Pubmed: 27438319.

20. Adamski P, Adamska U, Ostrowska M, et al. Evaluating current and emerging antithrombotic therapy currently available for the treatment of acute coronary syndrome in geriatric populations. Expert Opin Pharmacother 2018; 19(13): 1415-1425, doi: 10.1080/14656566.2018.1510487, indexed in Pubmed: 30132731

21. Li YH, Fang CY, Hsieh IC, et al. 2018 Expert Consensus on the Management of Adverse Effects of Antiplatelet Therapy for Acute Coronary Syndrome in Taiwan. Acta Cardiologica Sinica. 2018;34(3):201-10. Epub 2018/05/31. doi: 10.6515/acs.201805_34(3).20180302a. PubMed PMID: 29844641; PubMed Central PMCID. : PMCPMC5968336, doi: 10.6515/acs.201805 34(3).20180302a

22. Gaubert M, Laine M, Richard T, et al. Effect of ticagrelor-related dyspnea on compliance with therapy in acute coronary syndrome patients. Int J Cardiol. 2014; 173(1): 120-121, doi: 10.1016/j.ijcard.2014.02.028, indexed in Pubmed: 24612612

23. Cattaneo M, Faioni EM. Why does ticagrelor induce dyspnea? Thromb Haemost. 2012; 108(6): 1031-1036, doi: 10.1160/TH12-08-0547, indexed in Pubmed: 23070079.

24. Caldeira D, Pinto FJ, Ferreira JJ. Dyspnea and reversibility profile of P2Y ${ }_{12}$ antagonists: systematic review of new antiplatelet drugs. Am J Cardiovasc Drugs. 2014; 14(4): 303-311, doi: 10.1007/s40256-0140071-6, indexed in Pubmed: 24659260.

25. Minner SA, Simone P, Chung BB, et al. Successful Reversal of Bradycardia and Dyspnea With Aminophylline After Ticagrelor Load. J Pharm Pract. 2018; 31(1): 112-114, doi: 10.1177/0897190016680978, indexed in Pubmed: 27920235

26. Lindholm D, James S, Andersson J, et al. Caffeine and incidence of dyspnea in patients treated with ticagrelor. Am Heart J. 2018; 200: 141-143, doi: 10.1016/j.ahj.2018.02.011, indexed in Pubmed: 29898843. 